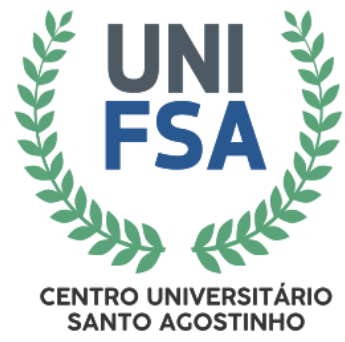

SANTO ACOSTINHO

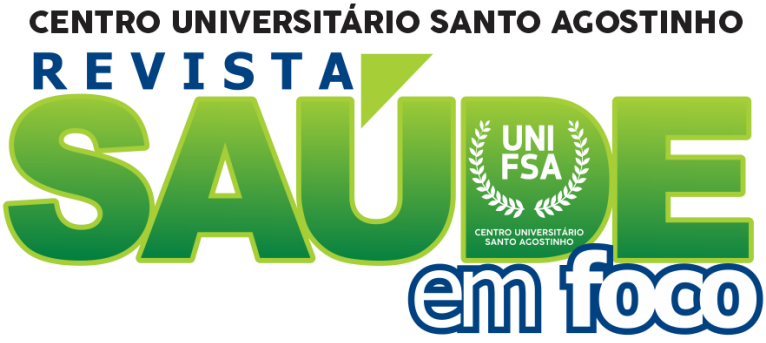

www4.fsanet.com.br/revista

Revista Saúde em Foco, Teresina, v. 8, n. 3, art. 1, p. 03-22, set./dez. 2021

ISSN Eletrônico: 2358-7946

http://dx.doi.org/10.12819/rsf.2021.8.3.1

\title{
Intervenções da Enfermagem nos Eventos Adversos da Transfusão Sanguínea: Perspectivas Reais
}

\author{
Nursing Interventions to Adverse Events of Blood Transfusion: Real Perspectives
}

Rejane Fernandes da Silva Vieira

Especialista em Hemoterapia e Terapia Celular, Faculdade Unyleya E-mail: rejfernandes25@gmail.com

Ivan Pricken de Bem

Mestre em Políticas Públicas em Saúde na Escola Fiocruz

E-mail: ivanprk@gmail.com

Milton Luiz Nascimento

Doutorando no Programa de Pós-graduação em Bioética na Universidade de Brasília Mestre em Bioética pela Universidade de Brasília Professor da Faculdade Unyleya, Distrito Federal E-mail: mylthon@ hotmail.com

Thalita Gomes do Carmo Doutora em Ciências do Cuidado em Saúde pelo Programa de Pós-Graduação da Universidade Federal Fluminense Professora adjunta da Universidade Federal Fluminense E-mail: thalitado@gmail.com

Aurélio Matos Andrade

Doutorando do Programa de Pós-Graduação em Ciências Médicas, Universidade de Brasília Mestre Profissional em Saúde Coletiva pela Universidade de Brasília

E-mail: aur87@hotmail.com

Endereço: Rejane Fernandes da Silva Vieira

Faculdade Unyleya. SIA trecho 17 , rua 10 , lote 455 . Zip code: 71.200-228 - Brasília/DF. Brasil.

Endereço: Ivan Pricken de Bem

Faculdade Unyleya. SIA trecho 17 , rua 10 , lote 455 . Zip code: 71.200-228 - Brasília/DF. Brasil.

Endereço: Milton Luiz Nascimento

Faculdade Unyleya. SIA trecho 17, rua 10, lote 455. Zip code: 71.200-228 - Brasília/DF. Brasil.

Endereço: Thalita Gomes do Carmo

Faculdade Unyleya. SIA trecho 17, rua 10, lote 455. Zip code: 71.200-228 - Brasília/DF. Brasil.

Endereço: Aurélio Matos Andrade

Faculdade Unyleya. SIA trecho 17, rua 10, lote 455. Zip

code: 71.200-228 - Brasília/DF. Brasil.
Editor-Chefe: Dr. Tonny Kerley de Alencar Rodrigues

Artigo recebido em 10/05/2021. Última versão recebida em 10/06/2021. Aprovado em 11/06/2021.

Avaliado pelo sistema Triple Review: a) Desk Review pelo Editor-Chefe; e b) Double Blind Review (avaliação cega por dois avaliadores da área).

Revisão: Gramatical, Normativa e de Formatação 


\section{RESUMO}

Identificar as intervenções da equipe de enfermagem frente aos eventos adversos da transfusão sanguínea nos serviços de hemoterapia. Método: Realizou-se uma revisão integrativa da literatura a partir do Check-list Preferred Reporting Items for Systematic Reviews and Meta-Analyses (PRISMA) e acrônimo foi estruturado em PICO, onde $\mathrm{P}=$ Profissionais da enfermagem, I= transfusão de sangue nos serviços de hemoterapia, $C=$ Não se aplica, $\mathrm{O}=$ Eventos adversos. As bases de dados consultados foram Medline via Pubmed, Scielo, Lilacs e BDENF, entre 2010 a 2020. Resultados: A análise foi feita em 14 artigos que evidenciaram: instrumentos de avaliação do conhecimento sobre o processo transfusional; monitoramento e adequação das atividades transfusionais; registro de identificação e dos procedimentos realizados nos serviços hemoterápicos; notificação dos sinais e sintomas dos pacientes; conduta do profissional da enfermagem nas possíveis reações adversas; normativas que interferem na prática da equipe de enfermagem; construção de protocolo de boas práticas antes e pós-transfusional; e a aplicação da atividade de educação permanente no processo transfusional. Discussão: A atuação de enfermeiros (as) e técnicos (as) de enfermagem nos serviços de hemoterapia são imprescindíveis para viabilizar a execução e o registro de todas as etapas dos procedimentos executados na transfusão de sangue e hemocomponentes, permitindo, dessa forma, a obtenção da rastreabilidade de reações transfusionais. Conclusão: As complicações associadas à transfusão de sangue precisam ser detectadas precocemente, por meio de protocolos assistenciais de enfermagem ao paciente que está sendo transfundido.

Palavras-Chave: Cuidados de Enfermagem. Serviço de Hemoterapia. Transfusão de Sangue. Reação Transfusional.

\section{ABSTRACT}

Objective: To identify the nursing team's interventions in the face of adverse blood transfusion events in hemotherapy services. Method: An integrative literature review was carried out using the Check-list Preferred Reporting Items for Systematic Reviews and MetaAnalyzes (PRISMA) and the acronym was structured in PICO, where $\mathrm{P}=$ Nursing professionals, $\mathrm{I}=$ blood transfusion in the services hemotherapy, $\mathrm{C}=$ Not applicable, $\mathrm{O}=$ Adverse events. The databases consulted were Medline via Pubmed, Scielo, Lilacs and BDENF, between 2010 and 2020. Results: The analysis was made in 14 articles that showed: instruments for assessing knowledge about the transfusion process; monitoring and adequacy of transfusion activities; registration of identification and procedures performed in blood therapy services; notification of patients' signs and symptoms; conduct of the nursing professional in possible adverse reactions; norms that interfere with the practice of the nursing team; construction of a good practice protocol before and after transfusion; and the application of permanent education activity in the transfusion process. Discussion: The performance of nurses and nursing technicians in hemotherapy services are essential to enable the execution and registration of all stages of the procedures performed in blood transfusion and blood components, thus allowing the obtaining of traceability of transfusion reactions. Conclusion: Complications associated with blood transfusion need to be detected early, using nursing care protocols for the patient being transfused.

Keywords: Nursing Care. Chemotherapy Service. Blood transfusion. Transfusion Reaction. 


\section{INTRODUÇÃO}

A transfusão de sangue humano tornou-se possível após a descoberta dos grupos sanguíneos pelo biólogo e médico austríaco Karl Landsteiner, em 1900 (JUQUEIRA, 2005). O uso do sangue com fins terapêuticos ganhou importância no suporte e no tratamento de hemorragias, em cirurgias, transplante de órgãos, entre outras indicações.

Apesar de ser uma terapia essencial, ela não é livre de riscos, sejam eles transfusionais como a complicação clínica por anafilaxia, hemolítica aguda, sobrecarga de volume. Erro humano na identificação de amostras de doador/receptor e técnica incorreta. Os riscos podem ser potencializados conforme as condições clínicas do paciente, transmissão de agentes infecciosos e o uso de equipamento inadequado (LOPES, 2008; BRASIL,2015).

Diante de tanta complexidade, todo o procedimento deve ser acompanhado por um corpo profissional especializado. A atuação da equipe de enfermagem nesse campo transfusional tem papel fundamental, pois sua presença assegura a execução de procedimentos seguros no manejo de terapias transfusionais e imprime socialmente a garantia de que os componentes do sangue sigam as normas técnicas vigentes dos órgãos reguladores (SILVA JÚNIOR, 2015).

No Brasil, o Ex-presidente Fernando Henrique Cardoso sancionou a Lei ${ }^{\circ} 10.205$, em 21 de março de 2001, que dispõe sobre a captação, proteção ao doador e ao receptor, com destaque à prevenção, ao diagnóstico e ao atendimento imediato das reações transfusionais adversas no âmbito das atividades hemoterápicas. Nesse sentido, a terapêutica transfusional se potencializa como um procedimento que salva vidas, cujo profissional da enfermagem tem participação no processo transfusional, assim como na compreensão dos riscos associados às complicações imediatas ou tardias (GURGEL, 2019).

A implementação das ações de vigilância em saúde das reações transfusionais subsidia a promoção da saúde e a prevenção de riscos no âmbito da hematologia e hemoterapia. Tendo isso em vista, é importante compreender as condutas da equipe de enfermagem em serviços de hemoterapia no contexto dos eventos adversos à transfusão de sangue, a fim de permitir uma reflexão sobre a prática profissional transfusional.

Entre as diretrizes básicas da boa prática transfusional estão: a) a indicação da transfusão feita pelo médico, baseada em critérios clínicos; b) a prescrição e a solicitação de transfusão sanguínea deve ser preenchida de forma que não cause dúvidas; c) checar atentamente a identificação do paciente; d) a identificação da amostra de sangue deve ser checada com cuidado; e) antes da transfusão, observar sinais vitais e investigar previamente a transfusão, os sinais e sintomas apresentados pelo paciente (hipertermia, vertigem e dispneia), essa medida 
ajuda a identificar possível reação transfusional; f) cuidado contínuo com o paciente politransfundido; g) o teste pré-transfusional não deve ser dispensado; h) nunca aproveitar o acesso para administrar medicação junto com a transfusão; i) administrar a transfusão lentamente; e j) não aquecer a bolsa de sangue em micro-ondas, estufa ou em banho-maria (NEVES, 2010).

É importante entender o conhecimento acerca das práticas em saúde da equipe de enfermagem em serviços de hemoterapia no contexto dos eventos adversos à transfusão de sangue, no sentido de promover o cuidado vinculado à terapêutica transfusional que, embora ofereça benefícios, não é isento de complicações que podem, até mesmo, acarretar em óbito. Este artigo teve por objetivo identificar as intervenções de enfermagem frente aos eventos adversos à transfusão de sangue nos serviços de hemoterapia.

\section{MÉTODO}

\subsection{Identificação do estudo}

Trata-se de um estudo de revisão integrativa de literatura que sintetizou resultados obtidos em pesquisas sobre intervenções de enfermagem em serviços de hemoterapia, de maneira sistemática, ordenada e abrangente. Essa revisão integrativa forneceu informações mais amplas transversais no contexto dos eventos adversos à transfusão de sangue ${ }^{(6)}$.

Utilizou-se a ferramenta Preferred Reporting Items for Systematic Reviews and MetaAnalyses (PRISMA - Statement), baseada em 7 principais etapas: construção de um protocolo de pesquisa; formulação da pergunta dentro da Prática Baseada em Evidência (PBE) utilizando o acrônimo PICO (Pacientes, Intervenção, Outcomes/Desfecho); definição dos descritores, estratégias de busca em cada uma das bases de dados selecionadas pelo pesquisador, devendo ser variadas; determinação, seleção e revisão dos critérios de inclusão e exclusão; avaliação crítica dos estudos; coleta de dados utilizando instrumentos que analisem em pares (dois pesquisadores simultaneamente); e síntese dos resultados/dados agrupados por semelhança (LIBERATI, 2009).

$\mathrm{Na}$ primeira etapa da revisão, os pesquisadores desenvolveram um protocolo de pesquisa, para levantamento de itens como: de que forma os estudos seriam encontrados, quais seriam os critérios de inclusão e exclusão dos artigos, para definição dos desfechos de interesse, verificação da acurácia dos resultados. 
Para atender a segunda etapa, construiu-se a pergunta de pesquisa, recorrendo-se à utilização da estratégia PICO, que representa um acrônimo para Pacientes, Intervenção, Comparação e Outcomes (Desfecho). Porém, nessa pesquisa, como não houve comparação, utilizou-se o PIO, onde o $\quad \mathrm{P}=$ Paciente/Problema, I=Intervenção), $\mathrm{O}=$ Outcome $/$ Resultado/Desfecho.

$\mathrm{P}$ - Profissionais da enfermagem

I - Transfusão de sangue nos serviços de hemoterapia

C- Não se aplica

$\mathrm{O}-$ Eventos adversos

Formulou-se, então, a seguinte pergunta de pesquisa: Quais são as intervenções de enfermagem frente aos eventos adversos à transfusão de sangue nos serviços de hemoterapia?

\subsection{Estratégia de busca}

A terceira etapa foi a elaboração da estratégia de busca com os tesauros obtidos na Medical Subject Headings (MeSH), Descritores em Ciências da Saúde (DECS), em: "Serviço de Hemoterapia", "transfusão de sangue" "cuidados de enfermagem" e "reação transfusional". Foi utilizado o operador booleano "AND" para termos distintos e "OR" para termos sinônimos, deixando a estratégia de busca mais sistemática e robusta.

Esta revisão foi realizada entre novembro de 2020 a janeiro de 2021 em três bases de dados científicas: 1) Literatura Latino America e do Caribe (Lilacs), 2) Scientific Library Online (SCIELO), 3) Medline via Pubmed e 4) BDENF.

\subsection{Critérios de elegibilidade}

Como critérios de inclusão foram selecionados artigos publicados em português, espanhol e inglês, que abordassem as temáticas sobre a equipe de enfermagem, segurança do sangue, cuidados em enfermagem, reação transfusional, com recorte temporal de 2010 a 2020. Buscas adicionais foram realizadas em sites institucionais a fim de identificar marcos regulatórios referentes à hemoterapia, buscando complementar as referências desta revisão. Foram excluídos os artigos que não continham informações relacionadas à prática da equipe de enfermagem no contexto da assistência hemotransfusional. 


\subsection{Seleção e análise de dados}

A coleta de dados foi realizada por meio da leitura criteriosa do título do artigo, resumos e palavras- chave de forma independente por dois dos autores (RFSV e AMA) com software Rayyan QRI@. As variáveis de extração foram autor, ano, país, tipo de intervenção prática, principais resultados, sendo estruturadas de forma independente por dois dos autores (RFSV e AMA) por meio do software Microsoft Excel@ 2016. As discordâncias foram analisadas por consenso.

A análise e a interpretação dos resultados foram feitas por meio da discussão sobre as práticas da equipe de enfermagem em serviços de hemoterapia, no contexto dos eventos adversos à transfusão, comparando-se os dados evidenciados na análise dos artigos selecionados ao referencial teórico, identificando lacunas do conhecimento em pauta, além da explicitação das conclusões e as implicações para da prática em hemotransfusão.

\subsection{Questões Éticas}

De acordo com as prerrogativas éticas da resolução 510/2016, o presente estudo foi dispensado do registro e da avaliação pelo sistema Comitê de Ética e Pesquisa (CEP) /Comissão Nacional de Ética em Pesquisa (CONEP), por se tratar de uma revisão com dados previamente coletados em estudos publicizados.

\section{RESULTADOS E DISCUSSÕES}

Foram identificados 33 artigos na Biblioteca Virtual em Saúde (BVS), em seguida, foram excluídas 9 publicações por duplicidade e 1 artigo por está indisponível para leitura completa. Na leitura de título e resumo 9 publicações foram excluídas: por não serem artigos primários, não tratar do contexto transfusional e da prática transfusional pela equipe de enfermagem. Dessa forma, foram elegíveis 14 artigos para análise qualitativa das evidências (Figura 1). 
Figura 1 - Fluxograma do processo de síntese de revisão

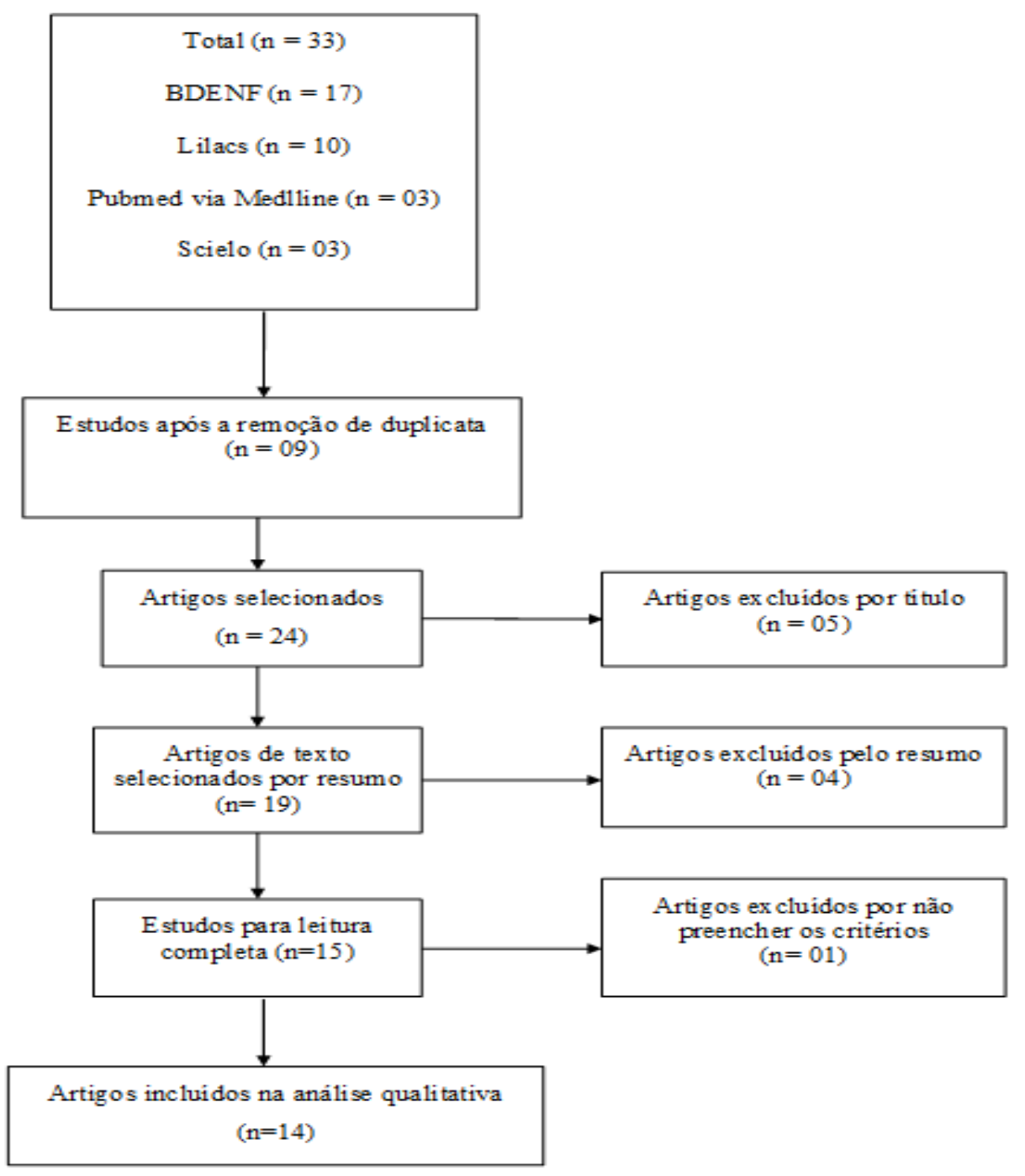

Fonte - Adaptação dos autores.

A maioria dos artigos selecionados foi publicada em 2016 (35,7\%). E, em relação ao delineamento dos estudos, grande parte utilizou abordagem qualitativa (42,8\%). Quanto aos objetivos, os estudos discutiram o conhecimento da equipe de enfermagem sobre o processo transfusional, as práticas de intervenção peritransfusionais com enfoque nas condutas e notificações frente às possíveis reações transfusionais (Quadro 1). 
Quadro 1. Caracterização dos artigos incluídos na revisão (n=14).

\begin{tabular}{|c|c|c|}
\hline Autor/ano & Tipo de estudo & Objetivo \\
\hline Torezan et al, 2010 & Estudo Transversal & $\begin{array}{l}\text { Avaliar o conhecimento dos enfermeiros sobre o } \\
\text { processo de cuidar do paciente que recebe } \\
\text { hemotransfusão. }\end{array}$ \\
\hline Souza et al, 2014 & $\begin{array}{l}\text { Pesquisa qualitativa } \\
\text { convergente } \\
\text { assistencial (PCA) }\end{array}$ & $\begin{array}{l}\text { Construir coletivamente, com os profissionais } \\
\text { de enfermagem, um instrumento de boas } \\
\text { práticas de cuidado a pacientes durante e após a } \\
\text { transfusão sanguínea. }\end{array}$ \\
\hline Tavares et al, 2015 & Estudo Transversal & $\begin{array}{l}\text { Verificar se há associação entre o conhecimento } \\
\text { dos profissionais de enfermagem sobre } \\
\text { hemotransfusão e as variáveis relacionadas aos } \\
\text { aspectos profissionais. }\end{array}$ \\
\hline Amaral et al, 2016 & Estudo Transversal & $\begin{array}{l}\text { Identificar o conhecimento de uma equipe de } \\
\text { enfermagem sobre o processo transfusional. }\end{array}$ \\
\hline Reis et al, 2016 & Estudo de Coorte & $\begin{array}{l}\text { Analisar o processo de registro do } \\
\text { monitoramento transfusional em um hospital } \\
\text { público de ensino. }\end{array}$ \\
\hline Cherem et al, 2016 & Pesquisa qualitativa & $\begin{array}{l}\text { Identificar o conhecimento dos enfermeiros } \\
\text { acerca dos cuidados de enfermagem no processo } \\
\text { pós-transfusional na unidade de terapia intensiva } \\
\text { neonatal. }\end{array}$ \\
\hline Mattia et al, 2016 & Pesquisa qualitativa & $\begin{array}{l}\text { Elaborar, juntamente com profissionais de } \\
\text { enfermagem, um instrumento de monitorização } \\
\text { do paciente submetido à transfusão sanguínea. }\end{array}$ \\
\hline Carneiro et al, 2017 & Estudo Transversal & $\begin{array}{l}\text { Verificar o conhecimento da equipe de } \\
\text { enfermagem sobre hemoterapia, reações } \\
\text { transfusionais imediatas e cuidados indicados } \\
\text { diante desses casos. }\end{array}$ \\
\hline Cherem et al, 2017 & Pesquisa qualitativa & $\begin{array}{l}\text { Analisar o conhecimento do enfermeiro acerca } \\
\text { do processo transfusional para o cuidado do } \\
\text { recém-nascido na unidade de terapia intensiva } \\
\text { neonatal }\end{array}$ \\
\hline \multirow[t]{2}{*}{ Silva et al 2017} & $\begin{array}{l}\text { Pesquisa } \\
\text { quantitativa }\end{array}$ & $\begin{array}{l}\text { Avaliar o conhecimento da equipe de } \\
\text { enfermagem diante das reações transfusionais } \\
\text { em um hospital do estado de Pernambuco. }\end{array}$ \\
\hline & Pesquisa qualitativa & $\begin{array}{l}\text { Analisar o conhecimento dos enfermeiros de } \\
\text { Unidade de Terapia Intensiva Neonatal sobre o }\end{array}$ \\
\hline
\end{tabular}




\begin{tabular}{|l|l|l|}
\hline Cherem et al, 2018 & processo de terapia transfusional. \\
\hline Forster et al, 2018 & Pesquisa qualitativa & $\begin{array}{l}\text { Conhecer a percepção dos enfermeiros quanto à } \\
\text { assistência de enfermagem no processo } \\
\text { transfusional. }\end{array}$ \\
\hline Nazário et al, 2019 & Métodos Mistos & $\begin{array}{l}\text { Identificar o conhecimento dos profissionais de } \\
\text { Enfermagem sobre a reação transfusional, antes } \\
\text { e após atividade de educação permanente. }\end{array}$ \\
\hline Buozi. et al, 2019 & Estudo Transversal & $\begin{array}{l}\text { Avaliar a adequação das atividades da } \\
\text { intervenção da Classificação das Intervenções } \\
\text { de Enfermagem Administração de } \\
\text { Hemoderivados para pacientes adultos. }\end{array}$ \\
\hline
\end{tabular}

Fonte: Elaboração dos autores

Dentre os tipos de intervenções, todas ocorreram no ambiente hospitalar, com destaque de $28,5 \%$ para Unidade Terapia Intensiva (UTI) adulta. E os principais resultados evidenciaram instrumentos de avaliação do conhecimento sobre o processo transfusional; monitoramento infeccioso e adequação das atividades transfusionais; registro de identificação e de procedimentos realizados nos serviços hemoterápicos; notificação dos sinais e sintomas dos pacientes; conduta do profissional da enfermagem nas possíveis reações adversas; normativas que interferem na prática da equipe de enfermagem em instituições hospitalares; construção de protocolo de Boas Práticas antes e pós-transfusional; e a aplicação da atividade de educação permanente com treinamento ou orientação na realização do processo transfusional (Quadro 2).

\section{Quadro 2 - Tipo de Intervenção e os principais resultados dos estudos selecionados} $(\mathbf{n}=14)$.

\begin{tabular}{|c|c|c|}
\hline Autor/ano & Tipo de intervenção & Principais resultados \\
\hline Torezan et al, 2010 & $\begin{array}{l}\text { Verificou-se o conhecimento de } \\
\text { enfermeiros sobre o processo de } \\
\text { hemotransfusão junto a pacientes } \\
\text { que recebem algum tipo de } \\
\text { hemoterápico. }\end{array}$ & $\begin{array}{l}\text { A maioria referiu está informada e } \\
\text { ter recebido treinamento referente } \\
\text { aos cuidados de enfermagem em } \\
\text { hemotransfusão, bem como } \\
\text { identificaram os sinais vitais } \\
\text { relacionados à transfusão e ao tempo } \\
\text { de infusão dos hemocomponentes. } \\
\text { Mais de } 80 \% \text { dos enfermeiros têm } \\
\text { conhecimento de como agir em caso } \\
\text { de reação transfusional causada por } \\
\text { hemólise. } \\
\text { Em se tratando de reconhecer os }\end{array}$ \\
\hline
\end{tabular}




\begin{tabular}{|c|c|c|}
\hline & & \begin{tabular}{lrrr} 
sinais e sintomas & das reações \\
transfusionais, apenas $42 \%$ dos \\
enfermeiros r & \multicolumn{2}{c}{ identificaram } \\
corretamente e & $27 \%$ & têm \\
conhecimento sobre compatibilidade \\
ABO/Rh.
\end{tabular} \\
\hline Souza et al, 2014 & $\begin{array}{l}\text { Discutiram-se os cuidados relatados } \\
\text { pela equipe de enfermagem com o } \\
\text { paciente crítico, realizados durante e } \\
\text { após a transfusão sanguínea para a } \\
\text { elaboração de um instrumento de } \\
\text { boas práticas transfusionais. }\end{array}$ & $\begin{array}{l}\text { Foi possível extrair das discussões } \\
\text { três ideias centrais: determinação da } \\
\text { velocidade de infusão, cuidados ao } \\
\text { término da infusão e condutas frente } \\
\text { às reações transfusionais. Essa } \\
\text { construção coletiva compôs o } \\
\text { instrumento de boas práticas dos } \\
\text { cuidados de enfermagem trans e pós- } \\
\text { transfusionais. }\end{array}$ \\
\hline Tavares et al, 2015 & $\begin{array}{l}\text { Investigou os fatores relacionados às } \\
\text { lacunas de conhecimento da equipe } \\
\text { de enfermagem na prática } \\
\text { transfusional. }\end{array}$ & $\begin{array}{l}\text { O conhecimento quanto à } \\
\text { importância dos cuidados } \\
\text { necessários em cada etapa } \\
\text { trasfusional foi de } 53,38 \% \text { na pré- } \\
\text { transfusional; na transfusional com } \\
51,25 \% \text {; e pós-transfusional } 62,68 \% \text {. } \\
\text { Apesar da metade dos profissionais } \\
\text { apresentarem déficit de } \\
\text { conhecimento em uma das etapas, } \\
\text { em sua maioria, 92,8\% dos } \\
\text { profissionais referiram segurança na } \\
\text { realização do processo transfusional. }\end{array}$ \\
\hline Amaral et al, 2016 & $\begin{array}{l}\text { Foram analisadas as respostas da } \\
\text { equipe de enfermagem quanto aos } \\
\text { cuidados com o paciente em } \\
\text { transfusão de hemocomponentes. }\end{array}$ & $\begin{array}{l}\text { Com resultado foi possível } \\
\text { identificar fragilidades quanto aos } \\
\text { conhecimentos relacionados aos } \\
\text { cuidados necessários durante a etapa } \\
\text { pré-transfusional e em caso de } \\
\text { reações transfusionais. }\end{array}$ \\
\hline Reis et al, 2016 & $\begin{array}{lll}\text { Identificou a qualidade } & \text { do } \\
\text { preenchimento dos formulários } & \text { de } \\
\text { monitoramento transfusionais. } & \end{array}$ & $\begin{array}{l}\text { Os formulários de monitoramento } \\
\text { apresentaram falhas de } \\
\text { preenchimento em } 53,4 \% \text {, quanto às } \\
\text { infusões que foram iniciadas após o } \\
\text { tempo preconizado corresponderam } \\
\text { a } 6 \% \text { e os formulários que não } \\
\text { apresentavam os sinais vitais dos } \\
\text { pacientes corresponderam a } 9,3 \% \text {. }\end{array}$ \\
\hline Cherem et al, 2016 & $\begin{array}{l}\text { Verificou-se o conhecimento de } \\
\text { enfermeiros acerca dos cuidados na } \\
\text { etapa pós-transfusional realizados } \\
\text { em recém-nascido em unidades de } \\
\text { terapia intensiva neonatal. }\end{array}$ & $\begin{array}{l}\text { Com resultado foi possível constatar } \\
\text { que as enfermeiras entrevistadas } \\
\text { reconheciam a importância de } \\
\text { realizar os cuidados pós- } \\
\text { transfusionais relacionados aos sinais } \\
\text { vitais, ao acesso venoso periférico, } \\
\text { ao descarte de bolas de sangue e dos } \\
\text { equipamentos usados na terapêutica. } \\
\text { Em contraposição, foi identificado } \\
\text { que as profissionais desconhecem a } \\
\text { necessidade do registro de } \\
\text { enfermagem previsto na legislação } \\
\text { que trata do assunto. }\end{array}$ \\
\hline
\end{tabular}




\begin{tabular}{|c|c|c|}
\hline Mattia et al, 2016 & $\begin{array}{l}\text { Discutiu-se sobre a qualidade dos } \\
\text { cuidados de enfermagem e } \\
\text { monitorização de pacientes } \\
\text { submetidos à transfusão sanguínea } \\
\text { para a elaboração de um instrumento } \\
\text { de monitoração a ser aplicado na } \\
\text { rotina. }\end{array}$ & $\begin{array}{l}\text { Foi possível extrair das discussões } \\
\text { com os profissionais as experiências } \\
\text { relacionadas à compreensão de: } \\
\text { qualidade dos cuidados de } \\
\text { enfermagem (conceitos, indicações e } \\
\text { reações transfusionais) e da } \\
\text { monitorização (identificação do } \\
\text { paciente e do hemocomponente, os } \\
\text { registros dos sinais vitais em todas as } \\
\text { etapas e observações } \\
\text { complementares). Essa construção } \\
\text { coletiva estruturou um instrumento } \\
\text { de monitorização com os registros } \\
\text { das informações sobre os pacientes } \\
\text { em transfusão sanguínea. }\end{array}$ \\
\hline Carneiro et al, 2017 & $\begin{array}{l}\text { Reconhecer e notificar reações } \\
\text { transfusionais precoces. }\end{array}$ & $\begin{array}{l}\text { Os sinais e sintomas das reações } \\
\text { transfusionais citados foram: febre } \\
(62,07 \%) \text {, seguida de prurido } \\
(44,83 \%) \text { e tremor }(37,93 \%) \text {. } \\
\text { Intervenção de enfermagem: } \\
\text { interromper a transfusão, comunicar } \\
\text { a equipe médica e comunicar o } \\
\text { banco de sangue. }\end{array}$ \\
\hline Cherem et al, 2017 & $\begin{array}{l}\text { Reconhecer e notificar reações } \\
\text { transfusionais precoces. }\end{array}$ & $\begin{array}{l}\text { Intervenção de enfermagem: } \\
\text { conhecer as reações transfusionais } \\
\text { nos neonatos, iniciar infusão de } \\
\text { forma lenta, realizar a transfusão em } \\
\text { no máximo } 4 \text { horas, suspender a } \\
\text { infusão em caso de reação adversa e } \\
\text { notificar equipe médica e banco de } \\
\text { sangue. }\end{array}$ \\
\hline Silva et al, 2017 & $\begin{array}{l}\text { Reconhecer reações transfusionais } \\
\text { precoces. }\end{array}$ & $\begin{array}{l}\text { Intervenção } \text { de } \\
\text { identificar o tempo máximo e } \\
\text { mínimo para a infusão do } \\
\text { concentrado de hemácias, avaliar } \\
\text { reações adversas, suspender a } \\
\text { infusão em caso de reação adversa e } \\
\text { notificar equipe médica e o banco de } \\
\text { sangue.. }\end{array}$ \\
\hline Cherem et al, 2018 & $\begin{array}{l}\text { Observar e checar a indicação da } \\
\text { terapia transfusional. }\end{array}$ & $\begin{array}{l}\text { Intervenção de enfermagem: atentar } \\
\text { para os exames de compatibilidade } \\
\text { transfusional, para a conferência da } \\
\text { bolsa de sangue, punção do acesso } \\
\text { venoso periférico e a não } \\
\text { administração de antibiótico no } \\
\text { momento da infusão, observar os } \\
\text { cuidados relativos à segurança na } \\
\text { administração do concentrado de } \\
\text { hemácia. }\end{array}$ \\
\hline Forster et al, 2018 & $\begin{array}{l}\text { Realizar o processo de enfermagem } \\
\text { antes, durante e após a transfusão } \\
\text { sanguínea. }\end{array}$ & $\begin{array}{l}\text { Intervenções de enfermagem: } \\
\text { Orientar os pacientes e familiares } \\
\text { sobre as possíveis reações adversas, } \\
\text { controlar sinais vitais, gotejamento } \\
\text { do concentrado de hemácia, manter } \\
\text { acesso venoso periférico exclusivo, }\end{array}$ \\
\hline
\end{tabular}




\begin{tabular}{|l|l|l|}
\hline & & $\begin{array}{l}\text { observar reações adversas, } \\
\text { interromper infusão ao menor sinal } \\
\text { de reação adversa, comunicar a } \\
\text { equipe médica ao banco de sangue. }\end{array}$ \\
\hline Nazário et al, 2019 & $\begin{array}{l}\text { Realizar educação da equipe de } \\
\text { enfermagem quanto as reações } \\
\text { adversas transfusionais. }\end{array}$ & $\begin{array}{l}\text { Intervenções de enfermagem: } \\
\text { Reconhecer reações adversas } \\
\text { imediatas, observar sinais vitais } \\
\text { comuns de reação adversa, atuar } \\
\text { frente a uma reação adversa do } \\
\text { paciente, reconhecer a diferença } \\
\text { entre sobrecarga volêmica e lesão } \\
\text { pulmonar relacionada à transfusão. }\end{array}$ \\
\hline Buozi. et al, 2019 & $\begin{array}{l}\text { Realizar o processo de enfermagem } \\
\text { baseado no Sistema de linguagem } \\
\text { padronizado antes, durante e após a } \\
\text { transfusão sanguínea. }\end{array}$ & $\begin{array}{l}\text { Intervenção de enfermagem: foram } \\
\text { elencadas 27 atividades descritas } \\
\text { pela Classificação das Intervenções } \\
\text { de Enfermagem, dentre essas, } \\
\text { algumas foram: verificar se o } \\
\text { hemoderivado foi preparado, tipado } \\
\text { e submetido à reação cruzada (se } \\
\text { aplicável) para o receptor; monitorar } \\
\text { os sinais vitais (p. ex., antes, durante } \\
\text { e após a transfusão); monitorar a } \\
\text { ocorrência de reações transfusionais; } \\
\text { evitar transfusão } \\
\text { de mais de uma unidade de sangue } \\
\text { ou hemoderivado de uma só vez, a } \\
\text { menos que isso seja necessário } \\
\text { devido à condição do receptor; e } \\
\text { administrar solução fisiológica ao } \\
\text { término da transfusão. }\end{array}$ \\
& &
\end{tabular}

Fonte: Elaboração dos autores

Dentre os resultados mais relevantes, os eventos adversos do ciclo do sangue, as reações transfusionais com situações de óbito, reação transfusional imediata (hemolítica aguda imunológica, lesão pulmonar relacionada à transfusão / Transfusion-Related Acute Lung Injury- TRALI, contaminação bacteriana), reação transfusional tardia com doença e reação hemolítica aguda imunológica, devem ser comunicadas ao serviço produtor do hemocomponente e à autoridade sanitária local nas primeiras 72 horas da ocorrência (BRASIL, 2015).

Sabe-se, também, que o evento adverso é um incidente que resulta em danos à saúde com necessidade de estabelecer estratégias para a segurança na prescrição, uso e administração de sangue e hemocomponentes, uma vez que a prática transfusional, embora traga benefícios não é isenta de riscos (BRASIL, 2013; SOBRAL, 2020). Dessa forma, são estabelecidas diretrizes para a prevenção, diagnóstico e atendimento imediato das reações transfusionais e adversas, pelo profissional que exerce a responsabilidade pela assistência 
direta ao paciente que está sendo transfundido (BRASIL, 2016), sendo atribuição dos (as) enfermeiros (as) e técnicos (as) de enfermagem seguir as normas quanto à execução, supervisão, administração e ao monitoramento da hemotransfusão , assumindo, dessa forma, papel fundamental na atuação e vigilância durante todo o processo transfusional (COFEN, 2006; ALBINI, 2010).

De acordo com Cherem et al (2016), a equipe de enfermagem demonstra conhecimento satisfatório em relação ao acompanhamento do recém-nascido nos primeiros dez minutos da infusão, apesar de não executarem passos importantes, conforme a legislação, tais como: a não conferência de todos os dados contidos no rótulo da bolsa de sangue, os sinais e sintomas diante de uma suspeita de reação transfusional, as condutas frente a cada reação, bem como o preenchimento da Ficha de Incidente Transfusional (FIT).

Quanto às intervenções da equipe de enfermagem relacionadas ao intra-procedimento transfusional, Reis et al (2016) observaram falhas quanto ao tempo para início da transfusão, onde 4,9\% das bolsas de hemocomponentes permaneceram mais de 30 minutos fora da geladeira antes de serem transfundidas, sendo que 1,1\% das bolsas permanecem por mais de 1(uma) hora erroneamente acondicionadas. Em aproximadamente 6,8\% das transfusões, não havia registro do horário de recebimento da bolsa e nem do início da infusão, não sendo possível identificar se foi iniciada no tempo recomendado, no entanto, é importante ressaltar que, as normas gerais de hemotransfusão orientam que a equipe de enfermagem deve assegurar que a transfusão seja iniciada nos trinta minutos após a remoção da bolsa do refrigerador do banco de sangue, não devendo o tempo máximo de infusão ultrapassar quatro horas (PAIVA, 2013).

Reis et al (2016) constataram erros no preenchimento do instrumento de monitorização, os quais não continham dados exigidos por lei para identificação criteriosa do paciente e informações para rastreabilidade do ato transfusional. Além disso, $15,7 \%$ dos pacientes apresentarem alguma alteração nos sinais vitais, apenas em 36,7\% houve registro das medidas terapêuticas tomadas, como administração de medicamento, interrupção da transfusão, comunicado ao médico responsável e adiamento da bolsa.

Nesse sentido, Cherem et al (2017) demonstraram que o profissional da enfermagem apresenta pouco conhecimento acerca da legislação quanto à aferição dos dados vitais ao término da infusão, além do que, não foram observados os dados referentes à necessidade de manter preventivamente o acesso venoso periférico. Estudo semelhante de Paiva et al (2013), demonstraram que $83,8 \%$ dos profissionais da enfermagem não aferiram sinais vitais no póstransfusional, ou então deixaram de anotar. Desse modo, se faz necessário conhecer 
amplamente os processos inerentes à transfusão sanguínea para a qualificação do cuidado minimizando riscos e complicações no tratamento de pacientes (SOUZA, 2014; BRASIL, 2013a).

É importante mencionar que a educação e atualização continuada em hemoterapia; a melhora dos protocolos operacionais padrão hemoterápicos contribuem para o aumento da segurança transfusional. Essas medidas também atuam no fortalecimento dos Comitês Transfusionais e na ressignificação das estruturas hospitalares que surgiram na década de 1980 do século XX, com a finalidade de garantir a correta utilização de sangue e hemoderivados (BRASIL, 2015; FORSTER, 2018).

Conforme Carneiro et al (2017), Forster et al (2018) e Tavares et al (2015), evidenciaram que os profissionais de enfermagem se sentem preparados para acompanhar o paciente durante a terapia transfusional, contudo, a maioria nunca participou de curso, treinamento e/ou capacitação/educação continuada na área de hemotransfusão. Tendo isso em vista, infere-se que são necessários conhecimentos aprofundados e a capacitação da equipe de enfermagem ao longo do tempo de exercício da profissão, reconhecendo a segurança assistencial (ALBINI, 2010).

$\mathrm{Na}$ abordagem dos profissionais da enfermagem intensivistas, Buozi. et al (2016) evidenciaram que a maioria das atividades da intervenção segundo a taxonomia da Nursing Interventions Classification (NIC) na administração de hemoderivado (administração de sangue ou hemoderivados e monitoramento da reação do paciente), apresentaram importância quanto à prevenção, vigilância e às ações ao longo do processo transfusional. Contudo, Torezan et al (2010), afirmam que muitos profissionais da enfermagem não observam os aspectos importantes da compatibilidade sanguínea dos sistemas $\mathrm{ABO} / \mathrm{Rh}$, falhas na conferência dos pedidos de exames, na etiquetação e identificação de hemocomponentes comprometem a qualidade nos serviços hemoterápicos (SOBRAL, 2020; BRASIL 2016).

A ausência de programas de educação continuada voltados à atuação da enfermagem em serviços de hemoterapia é uma realidade, tornando necessária a organização de programas de educação em serviço que desenvolvam conhecimentos, habilidades e competências do trabalho da enfermagem em seu cotidiano no banco de sangue (ALBINI, 2010; CHEREM,2016). Para Nazário et al (2019), a educação permanente em saúde pode proporcionar melhoria na assistência em saúde e, em se tratando de reações transfusionais, sua utilização é necessária para prevenir eventos adversos durante o atendimento e preparar os profissionais para atender e identificar os agravos na rotina laboral. 


\subsection{Limitações e Fortalezas}

As limitações desta revisão integrativa foram: 1) o baixo quantitativo de bases de dados investigadas; 2) restrição de local de estudo não possibilitando a extrapolação dos achados para outros países; 3) o baixo desenvolvimento de pesquisas de intervenção/ experimentais; 4) a falta de estudos que relacionem a variável raça/cor acerca da hemotransfusão avaliando a equipe de enfermagem; e 5) lacunas no conhecimento das práticas hemoterápicas ideais por parte dos profissionais de enfermagem. Além daqueles relacionados ao sistema de busca online, que são falhos.

As fortalezas consistem nos dados evidenciados sobre incidentes e eventos adversos decorrentes da hemotransfusão notificados ao Sistema Nacional de Vigilância Sanitária, a fim de prevenir o aparecimento ou a recorrência de efeitos (BRASIL, 2013b). Cabe salientar que todo serviço de hemoterapia deve ter um sistema para a detecção, notificação e avaliação das complicações transfusionais, que inclua procedimentos operacionais para a detecção, o tratamento e a prevenção das reações transfusionais (BRASIL, 2004).

\subsection{Implicações para prática}

Destaca-se a clara necessidade dos profissionais de enfermagem de terem educação permanente a respeito da prática transfusional, além de treinamento em serviço para uma melhor conduta mediante qualquer intercorrência que possa acontecer, visando um melhor atendimento e sem riscos maiores para o paciente (CHEREM, 2016).

Outra implicação para a prática é a atenção redobrada que deve ser aplicada na conferência da bolsa e do paciente à beira do leito, imediatamente antes da transfusão, conferindo seus dados com os dados do hemocomponente, avaliação criteriosa da indicação transfusional são recomendações na prevenção das reações transfusionais. Apesar de todos os cuidados utilizados, a transfusão ainda apresenta riscos - entre os quais, transmissão de doenças infecciosas, imunossupressão e aloimunização -, devendo ser realizada somente na em caso de indicação precisa (FLAUSINO, 2015). Dessa forma, a seleção pré-transfusional é uma operação que visa a escolher os hemocomponentes, com base em provas laboratoriais adequadas, das unidades de sangue e de hemocomponente.

Mediante as reações transfusionais, em geral, deve-se interromper imediatamente a transfusão, verificar os sinais vitais do paciente; comunicar o incidente ao médico assistente, instituir o tratamento clínico específico e preencher a ficha de notificação de reação 
transfusional, pois, de acordo com a RDC 34/2014, que normatiza as boas práticas para serviço de hemoterapia, determina que o serviço de hemoterapia deve ter procedimentos escritos para detecção, registro, comunicação e notificação dos eventos adversos à doação (NEVES, 2010; BRASIL,2014).

\section{CONSIDERAÇÕES FINAIS}

As complicações associadas à transfusão de sangue precisam ser prevenidas pela adoção de procedimentos padronizados pela equipe da assistência direta ao paciente que está sendo transfundido. Além disso, a segurança do processo transfusional demanda reconhecimento adequado das reações adversas inerentes ao processo, para direcionar intervenções de educação em saúde, junto às equipes de enfermagem que realizam de assistência direta, a fim de reduzir os riscos e minimizar os danos.

Nesse sentido, a atuação de enfermeiros (as) e técnicos (as) de enfermagem nos serviços de hemoterapia são imprescindíveis para viabilizar a execução e o registro de todas as etapas dos procedimentos executados na transfusão de sangue e hemocomponentes, permitindo, dessa forma, a obtenção da rastreabilidade de reações transfusionais. Sabe-se ainda que, os incidentes transfusionais devem ser sistematicamente identificados, diagnosticados, investigados, notificados e analisados.

É notório que a identificação das práticas em saúde da equipe de enfermagem contribui para a segurança transfusional, assim como para motivar estudos e reflexões acerca do aprimoramento da assistência hemoterápica. Observa-se ainda, a necessidade da construção e do conhecimento de protocolos que norteiem a prática transfusional no contexto das reações adversas, o reconhecimento adequado de sinais e sintomas frente às intercorrências transfusionais, promovendo a capacitação em atividades laborativas, com atitudes que minimizem complicações ao paciente.

É fundamental, também, priorizar as práticas e os conhecimentos específicos na área da hemoterapia para os profissionais de enfermagem, acerca das normatizações que regem o ato transfusional, e consequente execução adequada das etapas do processo de investigação e monitoramento das reações transfusionais. Dessa forma, recomenda-se que as instituições hemoterápicas, promovam educação e atualização continuadas acerca da qualificação transfusional, de modo a proporcionar a promoção da segurança transfusional. 


\section{REFERÊNCAIS}

BRASIL. Ministério da Saúde (MS). Guia para o uso de hemocomponentes [Internet]. 2015. p. 139. [cited 18 Abr 2021] Available from: https://pesquisa.bvsalud.org/portal/resource/pt/biblio-943014.

BRASIL. Agência Nacional de Vigilância Sanitária (Anvisa). Instrução Normativa 1, de 17 de março de 2015. Dispõe sobre os procedimentos, normas e diretrizes do sistema nacional de hemovigilância citados na Resolução da Diretoria Colegiada 34, de 11 de junho de 2014, que dispõe sobre as Boas Práticas no Ciclo do Sangue. [Acesso 18 Abr 2021] Available from: http://antigo.anvisa.gov.br/legislacao\#/.

BRASIL. Agência Nacional de Vigilância Sanitária (Anvisa). Resolução - RDC no 36, de 25 de julho de 2013. Institui ações para a segurança do paciente em serviços de saúde e dá outras providencias . [Resolução na Internet]. Diário Oficial [ da República Federativa do Brasil]. Brasília, 26 julho. 2013. [Acesso 18 Abr 2021] Available from: http://antigo.anvisa.gov.br/legislacao/?inheritRedirect=true\#/visualizar/29068.

BRASIL. Agência Nacional de Vigilância Sanitária (Anvisa). Resolução - RDC no 36, de 25 de julho de 2013. Institui ações para a segurança do paciente em serviços de saúde e dá outras providencias . [Resolução na Internet]. Diário Oficial [ da República Federativa do Brasil]. Brasília, 26 julho. 2013. [Acesso 18 Abr 2021] Available from: http://antigo.anvisa.gov.br/legislacao/?inheritRedirect=true\#/visualizar/29068.

BRASIL. Agência Nacional de Vigilância Sanitária (Anvisa). Resolução da Diretoria Colegiada $\mathrm{n}^{\mathrm{o}} 158$ de 4 de fevereiro de 2016. Redefine o Regulamento Técnico de Procedimentos Hemoterápicos. [Resolução na Internet]. Diário Oficial [ da República Federativa do Brasil]. Brasília,. 05 de Fev de 2016. [Acesso 18 Abr 2021] Disponível em: https://www.in.gov.br/web/dou/-/portaria-n-158-de-4-de-fevereiro-de-2016-22301274.

BRASIL. Agência Nacional de Vigilância Sanitária (Anvisa). Resolução da Diretoria Colegiada n ${ }^{\circ}$ 34, de 11 de junho de 2014. Dispõe sobre as Boas Práticas no Ciclo do Sangue. [Resolução na Internet]. Diário Oficial [ da República Federativa do Brasil]. Brasília, 16 de jun. 2014. [Acesso 18 Abr 2021] Disponível em: http://antigo.anvisa.gov.br/legislacao/?inheritRedirect=true\#/visualizar/29223.

BRASIL. Agência Nacional de Vigilância Sanitária (Anvisa). Resolução $n^{\circ} 153$, de 14 de junho de 2004. Determina o Regulamento Técnico para os procedimentos hemoterápicos,[...].[Resolução na internet].Diário Oficial [ da República Federativa do Brasil]. Brasília, 24 jun. 2004 [Acesso 18 Abr 2021] Disponível em: http://antigo.anvisa.gov.br/legislacao/?inheritRedirect=true\#/visualizar/26230.

COFEN. Conselho Federal de Enfermagem. Resolução COFEN n. 306, de 25 de abril de 2006. Normatiza a atuação do Enfermeiro em Hemoterapia. Rio de Janeiro: COFEN; 2006. [Acesso 18 Abr 2021] Disponível em: http://www.cofen.gov.br/resoluo-cofen3062006_4341.html. 
ALBINI, L.; LABRONICI, L. M.; LACERDA, M. R. Atuação da enfermeira em comitê transfusional. Cogitare enferm [Internet], v.15, n.3, p.556-9. 2010. [Acesso 18 Abr 2021] Disponível em: https://pesquisa.bvsalud.org/portal/resource/pt/lil-575849.

AMARAL, J. H. S et al. Hemoterapia: um desafio no cotidiano da equipe de enfermagem TT - Hemotherapy: a challenge in the daily team of the nursing team. Rev enferm UFPE line [Internet], v.10, n.6, p.4820-7. 2016. [Acesso 18 Abr 2021] Disponível em: http://www.revista.ufpe.br/revistaenfermagem/index.php/revista/article/view/8555/pdf_1994.

BUOZI, B. C et al. Adequação das atividades da intervenção "administração de hemoderivados" da classificação das intervenções de enfermagem para pacientes adultos. reme rev min enferm [Internet], v. 23, n.1, e-1258. 2019. [Acesso 18 Abr 2021] Disponível em: http://www.reme.org.br/exportar-pdf/1404/e1258.pdf.

CARNEIRO, V. S. M.; BARP, M.; COELHO, M. A. Hemoterapia e reações transfusionais imediatas: atuação e conhecimento de uma equipe de enfermagem. REME rev min enferm [Internet], v.21, n.1, p.1-8. 2018. [Acesso 18 Abr 2021] Disponível em: http://www.reme.org.br/exportar-pdf/1167/e1031.pdf.

CHEREM, E. O et al. Cuidado pós-transfusional na unidade de terapia intensiva. Rev baiana enferm [Internet], v. 30, n. 4, p. 1-8, out./dez. 2016 [Acesso 18 Abr 2021] Disponível em: https://portalseer.ufba.br/index.php/enfermagem/article/view/16338/pdf.

CHEREM, E. O et al. Processo de terapia transfusional em unidade de terapia intensiva neonatal: o conhecimento do enfermeiro. Context enferm [Internet], v. 27, n. 1, e1150016, 2018.

[Acesso 18 Abr 2021] Disponívelem: http://www.scielo.br/scielo.php?script=sci_arttext\&amp

CHEREM, E.O et al. Saberes do enfermeiro para o cuidado no processo transfusional em recém-nascidos. Rev Gauch Enferm [Internet], v.38, n.1, e63557-e63557. 2017. [Acesso 18 Abr 2021] Disponível em: http://www.scielo.br/scielo.php?script=sci_arttext\&amp

ERCOLE, F. F,; MELO, L. S.; ALCOFORADO, C. L. G. C. Revisão integrativa versus revisão sistemática. REME rev min enferm [Internet], v.18, n.1, p.9-11. 2014. [Acesso 18 Abr 2021] Disponível em: http://www.reme.org.br/exportar-pdf/904/v18n1a01.pdf.

FLAUSINO, G. F et al. O ciclo de produção do sangue e a transfusão: o que o médico deve saber. Rev méd Minas Gerais [Internet], v.25, n.2.p. 269-279. 2015. [Acesso 18 Abr 2021]Disponível em: http://rmmg.org/exportar-pdf/1784/v25n2a19.pdf.

FORSTER F et al. Percepção dos enfermeiros quanto à assistência de enfermagem no processo transfusional. Enferm foco [Internet], v.9, n.3, p.:71-5. 2018. [Acesso 18 Abr 2021]. Disponível em: http://revista.cofen.gov.br/index.php/enfermagem/article/view/1509/464

GURGEL, A. P et al. Paciente crítico: segurança em terapia transfusional mediante lista de verificações. Rev bras ciênc saúde [Internet], v.23, n.4, p.525-34.2019. [Acesso 18 Abr 2021]; Disponível em: https://periodicos.ufpb.br/ojs2/index.php/rbcs/article/view/37205/29198.

JUNQUEIRA, P. C.; ROSENBLIT, J.; HAMERSCHLAK, N. História da Hemoterapia no 
Brasil. Rev. Bras. Hematol. Hemoter. [Internet]. [Acesso 18 Abr 2021], v.27, n.3, p. 201-207. Set. 2005. Disponível em: http://www.scielo.br/scielo.php?script=sci_arttext\&pid=S1516$84842005000300013 \& \operatorname{lng}=\mathrm{en}$.

LIBERATI, A et al. The PRISMA statement for reporting systematic reviews and metaanalyses of studies that evaluate health care interventions: explanation and elaboration. PLoS Med. [Internet] v.6, n.7, e1000100. 2009. Disponível em: https://doi.org/10.1371/journal.pmed.1000100

LOPES, M. S. S. N.; PROIETTI, A. B. F. C. HTLV-1/2 transfusional e hemovigilância: a contribuição dos estudos de look-back. Rev bras hematol hemoter [Internet], v.30, n.3, p. 229-40. 2008. [Acesso 18 Abr 2021]Disponível em: http://www.scielo.br/scielo.php?script=sci_arttext\&amp

MATTIA, D.; ANDRADE, S. R. Cuidados de enfermagem na transfusão de sangue: um instrumento para monitorização do paciente. Texto Contexto Enferm, v.25, n.2, abr-jun 2016. Disponible en <http://www.index-f.com/textocontexto/2016/25209p.php> Consultado el 24 de Marzo de 2021.

NAZÁRIO, S. S et al. Educação permanente de equipe de enfermagem em reação transfusional. Rev enferm UFPE line [Internet], v.13, n.2, p.307-14.2019. [Acesso 18 Abr 2021]. Disponível https://periodicos.ufpe.br/revistas/revistaenfermagem/article/view/237361/31272

NEVES, M. S. A.; DELGADO, R. B. Suporte hemoterápico ao paciente em emergência. Rev méd Minas Gerais [Internet],v.20, n.4, p.: 568-577. 2010. [Acesso 18 Abr 2021]. Disponível em: http://rmmg.medicina.ufmg.br/index.php/rmmg/article/view/319/305.

PAIVA, S. S et al. Avaliação dos registros de enfermagem em hemoterapia de um hospital geral Av enferm [Internet], v.31, n.1, p.103-12. 2013. [Acesso 18 Abr 2021]. Disponível em: http://www.scielo.org.co/scielo.php?script=sci_arttext\&amp.

REIS, V. N et al. transfusional: análise da prática assistencial em um hospital público de ensino. Einstein (Säo Paulo) [Internet], v.14, n.1 ,p.41-6.2016. [Acesso 18 Abr 2021]. Disponível em: http://www.scielo.br/scielo.php?script=sci_arttext\&amp.

SILVA, E. M et al. Desafios da enfermagem diante das reações transfusionais. Rev enferm UERJ [Internet], v. 25, e11552-e11552. 2017. [Acesso 18 Abr 2021]. Disponível em: http://www.e-publicacoes.uerj.br/index.php/enfermagemuerj/article/view/11552/22666.

SILVA JÚNIOR J. B.; COSTA, C. S.; BACCARA, J. P. A. Regulação de sangue no Brasil: contextualização para o aperfeiçoamento. Rev Panam Salud Publica [Internet].; v.38, n.4, p.333-338, 2015. [Acesso 18 Abr 2021]. Disponível em: https://iris.paho.org/handle/106652/18383.

SOBRAL, P. A. S.; GÖTTEMS, L. B. D.; SANTANA, L. A. Hemovigilância e segurança do paciente: análise de reações transfusionais imediatas em idosos. Rev bras enferm [Internet]. v.73, (supl.3),e20190735-e20190735. 2020. [Acesso 18 Abr 2021]. Disponível em: http://www.revenf.bvs.br/scielo.php?script=sci_arttext\&amp 
SOUZA, G. F et al. Boas práticas de enfermagem na unidade de terapia intensiva: cuidados durante e após a transfusão sanguínea. REME rev min enferm [Internet].v. 18, n.4, p.939-46. 2014. [Acesso 18 Abr 2021]

TAVARES, J. L et al. Fatores associados ao conhecimento da equipe de enfermagem de um hospital de ensino sobre hemotransfusão. Rev latino-am enferm [Internet]. v.23, n.4, p.595602. 2015. [Acesso 18 Abr 2021] Disponível em: http://www.scielo.br/scielo.php?script=sci_arttext\&amp

TOREZAN, G.; SOUZA, E. N. Transfusão de hemoderivados: os enfermeiros estão preparados para o cuidado peritransfusional? Rev enferm UFPE online [Internet], v.4, n.2, p.658-65. 2010 [Acesso 18 Abr 2021]. Disponível em: http://www.ufpe.br/revistaenfermagem/index.php/revista/article/view/845/pdf_52

\section{Como Referenciar este Artigo, conforme ABNT:}

VIEIRA, R. F. S; BEM, I. P; NASCIMENTO, M. L; CARMO, T. G. Intervenções da Enfermagem nos Eventos Adversos da Transfusão Sanguínea: Perspectivas Reais. Rev. Saúde em Foco, Teresina, v. 8, n. 3, art. 1, p. 03-22, set./dez 2021.

\begin{tabular}{|l|c|c|c|c|}
\hline \multicolumn{1}{|c|}{ Contribuição dos Autores } & $\begin{array}{l}\text { R. F. S. } \\
\text { Vieira }\end{array}$ & $\begin{array}{l}\text { I. P. } \\
\text { Bem }\end{array}$ & $\begin{array}{l}\text { M. } \\
\text { Nascimento }\end{array}$ & $\begin{array}{l}\text { T. G. } \\
\text { carmo }\end{array}$ \\
\hline 1) concepção e planejamento. & $\mathrm{X}$ & & & \\
\hline 2) análise e interpretação dos dados. & $\mathrm{X}$ & $\mathrm{X}$ & $\mathrm{X}$ & $\mathrm{X}$ \\
\hline 3) elaboração do rascunho ou na revisão crítica do conteúdo. & $\mathrm{X}$ & $\mathrm{X}$ & $\mathrm{X}$ & $\mathrm{X}$ \\
\hline 4) participação na aprovação da versão final do manuscrito. & $\mathrm{X}$ & $\mathrm{X}$ & $\mathrm{X}$ & $\mathrm{X}$ \\
\hline
\end{tabular}

